\title{
SprachGeschichten mit Migrationshintergrund: demografische und biografische Perspektiven auf Sprachkenntnisse und Spracherleben
}

\begin{abstract}
Um der zunehmenden „diversification of diversity“, die die sozialen Verhältnisse vor allem in vielen westlichen Großstädten kennzeichnet, gerecht zu werden, wird seit einiger Zeit der Begriff „Superdiversität“ verwendet. In diesem Zusammenhang haben sich sozialwissenschaftliche Untersuchungen vornehmlich mit unterschiedlichen Migrationsmustern bzw. -praktiken befasst, wobei verschiedene soziale Dimensionen im Mittelpunkt stehen. Die sprachliche Diversität als Merkmal dieses Phänomens wurde bis jetzt aber relativ wenig beachtet. Allerdings bieten so genannte „home language surveys“ in bestimmten deutschen Städten Einblicke in die Komplexität der sprachlichen Zusammensetzung einzelner urbaner Gesellschaften. Weder auf nationaler Ebene noch für die Bundeshauptstadt Berlin aber liegen amtliche Statistiken über die Sprachkenntnisse von Migranten in Deutschland vor.

In Wien und in London dagegen wurden anhand groß angelegter Erhebungen umfassende Daten über die in der österreichischen bzw. britischen Hauptstadt verwendeten Familiensprachen gesammelt und im Falle Londons sogar kartografisch dargestellt. Doch auch solche umfangreichen und ausführlichen Untersuchungen vermitteln nur einen Teilaspekt der sprachlichen Superdiversität: Schließlich geht es um mehr als Zahlen. In diesem Beitrag wird also zuerst ein kritischer Blick auf den gegenwärtigen Stand der Datenlage im Bereich der Sprachkenntnisse von Migranten in Deutschland geworfen. Anschließend wird vorgeschlagen, dass diese quantitative, demografische Perspektive durch einen qualitativen, biografischen Ansatz ergänzt werden kann. Im Sinne von Busch (2010) wird dabei exemplarisch das Spracherleben von zwei Bewohnern eines Berliner Mietshauses untersucht. Diese Analyse einzelner „SprachGeschichten“ ist somit auch eine Antwort auf den Aufruf von Gogolin (2010), die „sprachliche Textur von Migrationsgesellschaften“ näher zu untersuchen.
\end{abstract}

\section{Einführung}

In ihrer Einführung zu einer kürzlich erschienenen Sonderausgabe der Zeitschrift für Erziehungswissenschaft verweisen Ingrid Gogolin und Meinert Meyer (2010, S. 525) auf veränderte Migrationsformen und -muster in den vergangenen Jahrzehnten, die auch ,eine veränderte sprachliche Textur der Migrationsgesellschaften" hervorgebracht haben. In ihrem eigenen Artikel pflichtet Ingrid Gogolin (2010) Jan Blommaert (2010) und anderen (siehe z.B. Blommaert/Rampton 2011) bei, die sich dafür aussprechen, das Konzept der Superdiversität als Rahmen zu nutzen, um genau diese sprachliche 
Textur analytisch zu erfassen. Laut Gogolin wird Mehrsprachigkeit in Europa noch immer als exotisches Phänomen betrachtet, und wir wissen viel mehr über Mehrsprachigkeit in anderen Teilen der Welt (zum Beispiel, dass im Amazonas-Becken über 600 Sprachen gesprochen werden).

Was [aber] wissen wir über die Anzahl der Sprachen und ihrer Sprecher, die in einem Hochhaus in einer deutschen Großstadt existieren? Wie viele und welche Sprachen - neben dem Deutschen - werden von Schülerinnen und Schülern in Deutschlands Schulen und Hochschulen gesprochen? Auf wie viele und welche Sprachen müssen eine Sozialbehörde oder eine Klinik gefasst sein, wenn sie sichergehen wollen, dass die Kommunikation mit ihrer Klientel so störungsfrei wie möglich vonstattengeht? (Gogolin 2010, S. 530; siehe auch Meyer 2011)

Eine solche Darstellung ist in der Tat eine Herausforderung, und ich möchte dies als den Ausgangspunkt für meinen Beitrag nehmen. Ich möchte darauf eingehen, was wir scheinbar über die Zahl der Sprecher verschiedener Sprachen in deutschen Städten wissen, und was wir daraus ablesen können, oder auch nicht, und ich möchte versuchen, das Konzept der Superdiversität ganz speziell auf die komplexen und vielschichtigen soziolinguistischen Konstellationen im heutigen Deutschland anzuwenden.

Ich möchte jedoch mehr auf die einzelnen Sprecher und ihre sprachlichen Ressourcen eingehen, als auf die Sprachen, und auch weniger auf Sprachkenntnisse, als auf die Erfahrungen mit Sprache, angelehnt an Brigitta Buschs Konzept des „Spracherlebens“:

Mit dem Begriff Spracherleben umreißen wir einen Ansatz, der danach fragt, wie Menschen in mehrsprachigen Lebenszusammenhängen ihre Sprachlichkeit wahrnehmen und bewerten und welche Erfahrungen, Gefühle oder Vorstellungen sie damit verbinden. Oder andersherum gesagt: wie sie sich - gegenüber anderen oder sich selbst - in ihrer Mehrsprachigkeit erfahren, positionieren und darstellen. Gefragt wird nach dem Bezug des Spracherlebens zur individuellen Lebensgeschichte einerseits, zu historisch-gesellschaftlichen Konfigurationen mit ibren Zwängen, Machtgefügen, Diskursformationen und Sprachideologien andererseits. (Busch 2010, S. 58; Hervorhebung von mir)

Buschs Herangehensweise legt eine Perspektive nahe, die sich auf den Sprecher und seine Biografie einlässt und sich nicht mit „Sprachen“, sondern mit ,sprachlichen Ressourcen“ befasst, und wie diese genutzt werden, bzw. wie ihre Nutzung eingeschränkt wird. Diese Betrachtungsweise erlaubt uns, die Spuren nachzuvollziehen, wie diese sprachlichen Ressourcen im Laufe eines Lebens erworben, vertieft oder aufgegeben werden (siehe Blommaert/ Backus 2011). Und dies wiederum folgt Blommaerts Verständnis von „soziolinguistischem Repertoire":

It is tied to an individual's life and it follows the peculiar biographical trajectory of the speaker. When the speaker moves from one social space to another, his or her repertoire is affected, and the end result is something that mirrors, almost like an autobiography, the erratic lives of people. (Blommaert 2008, S. 16; Hervorhebung von mir) 
Busch argumentiert, dass dieses Herangehen eine besondere Zuwendung auf den einzelnen Menschen erfordert:

Ausgangspunkt sind nicht voretablierte Kategorisierungen, Abstraktionen und Quantifizierungen, sondern die Lebenswelt. Diese Lebenswelt, die - übertragen auf das Spracherleben - immer eine heteroglossische ist, wird nicht als statischer Hintergrund verstanden, sondern als dynamischer Horizont, als kollektiver intersubjektiver Pool der Wahrnehmungen, als gemeinsames Erfahrungsfeld und Feld transformierender Handlungen. (Busch 2010, S. 58)

Andere Beiträge in diesem Band erforschen die Beschaffenheit dieser „heteroglossischen Lebenswelt", die einzelne Menschen im heutigen Deutschland bewohnen (z.B. die Beiträge von Peter Auer, Ben Rampton und Heike Wiese). Die Fragen, auf die ich eingehen möchte, sind diese:

- Welche Art „transformierender Handlungen“ sind mit den Lebenswelten einzelner Menschen verbunden, und

- Wie kann eine biografische Untersuchung des Spracherlebens einzelner Menschen zu einer Soziolinguistik der Superdiversität beitragen?

Ich möchte beginnen, indem ich kurz auf einige Merkmale der Superdiversität eingehe, und dann werde ich - ebenfalls kurz - veranschaulichen, was wir über das Ausmaß der sprachlichen Superdiversität in Deutschland in quantitativer Hinsicht wissen, und die Grenzen dieses Wissens aufzeigen. Schließlich werde ich mehr im Detail darauf eingehen, welche Fragen wir daher angehen sollten, um diese statistischen Daten zu ergänzen, und was wir von den Sprachbiografien über die Sprachwelten lernen können, die die Menschen bewohnen.

\section{Superdiversität und ihre soziolinguistische Ausprägung}

Steven Vertovec $(2007,2010)$ hat verschiedentlich argumentiert, dass das Konzept der Superdiversität dem Prozess Rechnung tragen müsse, das er die „Diversifizierung der Diversität“ (Vertovec 2007, S. 1025) nennt und das viele der hochentwickelten Gesellschaften kennzeichnet, vor allem die großen Metropolen. Dabei gehen die Veränderungen, die solche Gesellschaften in den letzten Jahren erfahren haben, über den bloßen Anstieg der Zahlen von Einwanderern oder Herkunftsländern hinaus; sie sind vielmehr von immer vielschichtigeren und miteinander vernetzten Dimensionen von Diversität gekennzeichnet, die nicht nur verschiedene ethnische Identitäten, soziale Schichten, Kasten oder religiöse Zugehörigkeiten beinhalten, sondern auch, zum Beispiel, „differential immigration statuses and their concomitant entitlements and restrictions of rights, divergent labour market experiences, discrete gender and age profiles, patterns of spatial distribution, and mixed local area responses by service providers and residents" (ebd.). 
Außerdem überlagern diese neuen Schichten von Diversität bestehende - in einigen Fällen sehr lange bestehende - soziale Schichtungen. Dieser Prozess hat höchst komplexe „globale Nachbarschaften“ (Blommaert 2010, S. 7) und neue Konstellationen durch eine höhere „Dichte der Diversität“ (Mac Giolla Chríost 2007, S. 13) hervorgebracht. Zum Beispiel stehen Türken in Berlin in einem vielschichtigen Netzwerk sozialer Beziehungen: Sie haben Teil an begrenzten lokalen Interaktionen, aber sie nehmen auch translokale und transnationale Kontakte auf, indem sie reisen und in verschiedenen sozialen Medien kommunizieren (Skype, Internetforen, SMS und billige internationale Telefonverbindungen). Dieses Bild wird noch komplizierter, wenn man die wachsende Diversität verschiedener Aspekte von Migration betrachtet: Kategorien, Status, geschichtliche Voraussetzungen, „Ankunftsszenarien“ (Valentine/Sporton/Bang Nielsen 2009, S. 12) und Migrationsmuster (siehe Block 2006, S. 6-14).

Diese vielschichtigen Ausprägungen stehen in deutlichem Kontrast zur Verdichtung oder Tilgung kultureller Unterschiede in den öffentlichen Diskursen. Zum Beispiel verbirgt der offizielle Verweis auf Asylsuchende „Türken“ oder „Iraker" (also in Bezug auf ihr Herkunftsland) die Tatsache, dass viele oder gar die Mehrheit dieser Menschen Kurden sind. Derselbe diskursive Prozess vollzieht sich oft in Stadtteilen durch die „re-Territorialisierung" zerstreuter Gruppen oder Individuen (Blommaert 2008, S. 18), was dazu führt, dass sich dichte, ethnisch geprägte Nachbarschaften herausbilden, zum Beispiel „Bangladeshi Tower Hamlets“ in London, oder die „Polnische Thermometersiedlung“ in Berlin.

Daher ist es ein komplexes und methodisch herausforderndes Unterfangen, das Ausmaß der Diversität in den heutigen urbanen Gesellschaften, was die Ethnizität und Migrationsmuster betrifft, bestimmen zu wollen. Wenn man dieser Mischung dann noch Sprachen hinzufügt, erzeugt das erheblich viele weitere Schichten von Komplexität. Sprache ist eine der Dimensionen, die laut Vertovec zur Superdiversität beitragen, und die wachsende Zahl von Studien zu Ethnolekten und interaktionalen Praktiken wie „translanguaging“" veranschaulichen, welch breites Ausmaß eine „Soziolinguistik der Superdiversität" haben könnte (siehe z.B. Androutsopoulos 2007; Cheshire et al. 2011; Deppermann 2007; Freywald et al. 2011; Keim 2007; Pennycook 2010). Ein übergreifendes Thema ist jedoch, ob, und wenn ja, wie ein solches Forschungsfeld Fragen nach analytischen Ebenen (,scales") beinhalten kann (Collins/Slembrouck/Baynham (Hg.) 2009): Können wir, zum Beispiel, Zusammenhänge erfassen zwischen der Forschung zu spezifischen Sprachpraktiken einerseits und zur Verteilung von Sprachvarietäten und ihrer Sprecher andererseits?

Was wissen wir also über das Ausmaß der sprachlichen Diversität im heutigen Deutschland, und wie wird sie in den öffentlichen Diskursen dargestellt? 


\section{3. Über das Zählen und kartografische Erfassen von Sprachen in städtischen Kontexten: Sprachliche Diversität in Deutschland}

Es ist ja weithin anerkannt, dass jeder Versuch, die Anzahl der Sprachen, die in einer bestimmten Gesellschaft oder Gemeinschaft gesprochen werden, zu erfassen, unweigerlich mit schweren methodischen Problemen fertig werden muss (Barni/Extra (Hg.) 2008; Extra/Gorter (Hg.) 2001; Extra/ Yağmur (Hg.) 2004). Das ist sogar dann der Fall, wenn wir von voneinander abgrenzbaren Sprachen ausgehen, was manche als eine bequeme Fiktion betrachten (Makoni/Pennycook (Hg.) 2007). Doch ist einer der herausragendsten Aspekte der Sprach(en)politik in Deutschland, dass solche wissenschaftlichen Probleme die nationalen oder örtlichen Behörden nicht wesentlich beschäftigen: entweder weil sie die Frage nach den Sprachen im Zuge der Datensammlung zu ihrer jeweiligen Bevölkerungsgruppe gar nicht stellen, oder weil sie sich scheinbar mit der Gleichsetzung von Sprache und Herkunftsland zufriedengeben (vielleicht kann eine Regierung auch nur ein bestimmtes Maß an Diversität (v)ertragen). Während zum Beispiel Statistiken zu religiösen Einstellungen, Ehestand, Bildungsgrad, Beschäftigung und dem Herkunftsland der Eltern erstellt werden, fragt weder die neueste Volkszählung (www.zensus2011.de/fragebogen/haushalte-und-wiederholungs befragung.html) noch der Mikrozensus (Statistisches Bundesamt (Hg.) 2011) nach Sprachkenntnissen und -gebrauch. ${ }^{1}$

Sprachkenntnisse werden als statistische Kategorie erst im Verhältnis zu Bildung relevant (Klieme et al. (Hg.) 2010), wie auch in Studien zu Integration und sozialer Eingliederung. In ihrer weitreichenden Analyse erhältlicher Forschungsdaten zur „sprachlichen Integration“ in Deutschland stellt Haug (2008) fest, dass es keinerlei offizielle Daten über Sprachkenntnisse von Migranten gibt. Bestenfalls kann man eine Teilansicht auf der Basis verschiedener Tests zu Sprachvermögen und Selbstkategorisierungen in Umfragen erstellen.

„Home language surveys“ - also Erhebungen zu Sprachen, die „zu Hause" gesprochen werden -, im Rahmen von Forschungsprojekten durchgeführt, bieten eine viel detailliertere und differenziertere Darstellung, obwohl es davon bislang nur wenige gibt. Einzelne Forschungen, zum Beispiel das SPREEG Projekt in Essen (Chlosta/Ostermann 2005, 2006; Projektgruppe SPREEG 2001) und die FreiSprachen Studie in Freiburg (Decker/ Schnitzer 2011; www.freidaz.de) ergänzen die bahnbrechenden Erhebungen in Hamburg (Fürstenau/Goglin/Yağmur 2003) und Wien (Brizić/Lo Hufnagl 2011), die im Rahmen des „Multilingual Cities Project“ (Extra/Yağmur

Für Österreich liegen Daten zu Sprachkenntnissen vor (siehe Statistik Austria (Hg.) 2002), aber aus Platzgründen wird die Diskussion hier auf Deutschland begrenzt. 
(Hg.) 2004) beispielhafte Analysen der komplexen Dimensionen mehrsprachiger Praktiken liefern. An deren Forschungsstringenz und soziolinguistisches Verständnis reichen die Studien einzelner städtischer Behörden jedoch nicht heran.

Zahlen von Einwanderern nach Herkunftsland werden allerdings sowohl auf Bundes- als auch auf Länderebene (Statistisches Bundesamt (Hg.) 2006, 2011; Ministerium für Schule und Weiterbildung des Landes Nordrhein-Westfalen 2011) routinemäßig erhoben. Aber es ist natürlich sehr problematisch, von diesen Zahlen auf einzelne Sprachen (und ihre Sprecher) zu schließen: Katharina Brizić und Kutlay Yağmur (2008, S. 249) zum Beispiel erstellten eine Liste von 43 Sprachen, die in den letzten 20 Jahren in Umfragen in der Türkei erfasst wurden. Die Hälfte dieser Sprachen umfasst jeweils über 10.000 Sprecher in der Türkei. Chlosta/Ostermann (2005) treffen den Nagel auf den Kopf: „Warum fragt man nach der Herkunft, wenn man die Sprache meint?" Wenn die Frage nach der Sprache jedoch in offiziellen Umfragen gestellt wird, geht es weniger darum, was Kinder benötigen, um ihre ,anderen“ Sprachen zu lernen, als um die Notwendigkeit, die Ressourcen auf die auszurichten, die scheinbar besondere Unterstützung in Deutsch benötigen. In Berlin gibt es zum Beispiel genaue Daten über die Anzahl der Kinder, deren Muttersprache nicht Deutsch ist, aber alle diese werden unter der Kategorie „SchülerInnen nicht-deutscher Herkunftssprache" (ndH) zusammengefasst (siehe Ohliger/Raiser 2005): $\mathrm{Zu}$ einzelnen Sprachen gibt es keine Zahlen.

Das Amt für Statistik Berlin-Brandenburg hat zwar die Verteilung von Einwanderern nach Herkunftsland über die verschiedenen Bezirke von Berlin ermittelt (Rockmann 2010). Doch ohne Daten zu Sprachen in der Stadt ist es zurzeit unmöglich, die sprachliche Zusammensetzung einzelner Bezirke und die Verteilung von Sprachen über die Stadt auf eine Art und Weise darzustellen, wie es das „Language Capital Project“ in London getan hat. Dieses ehrgeizige Projekt versammelte die Angaben zu Familiensprachen von über einer Million Schülern, die 233 Sprachen nannten, von denen wiederum 30 von mehr als 1.000 Teilnehmern genannt wurden. Die Ergebnisse wurden in einer Serie von Karten veröffentlicht, die eine bemerkenswerte bildliche Darstellung der sprachlichen Superdiversität im heutigen London sowie beeindruckende Muster örtlicher Konzentration und Verdichtung eröffnen (Eversley et al. 2010). 


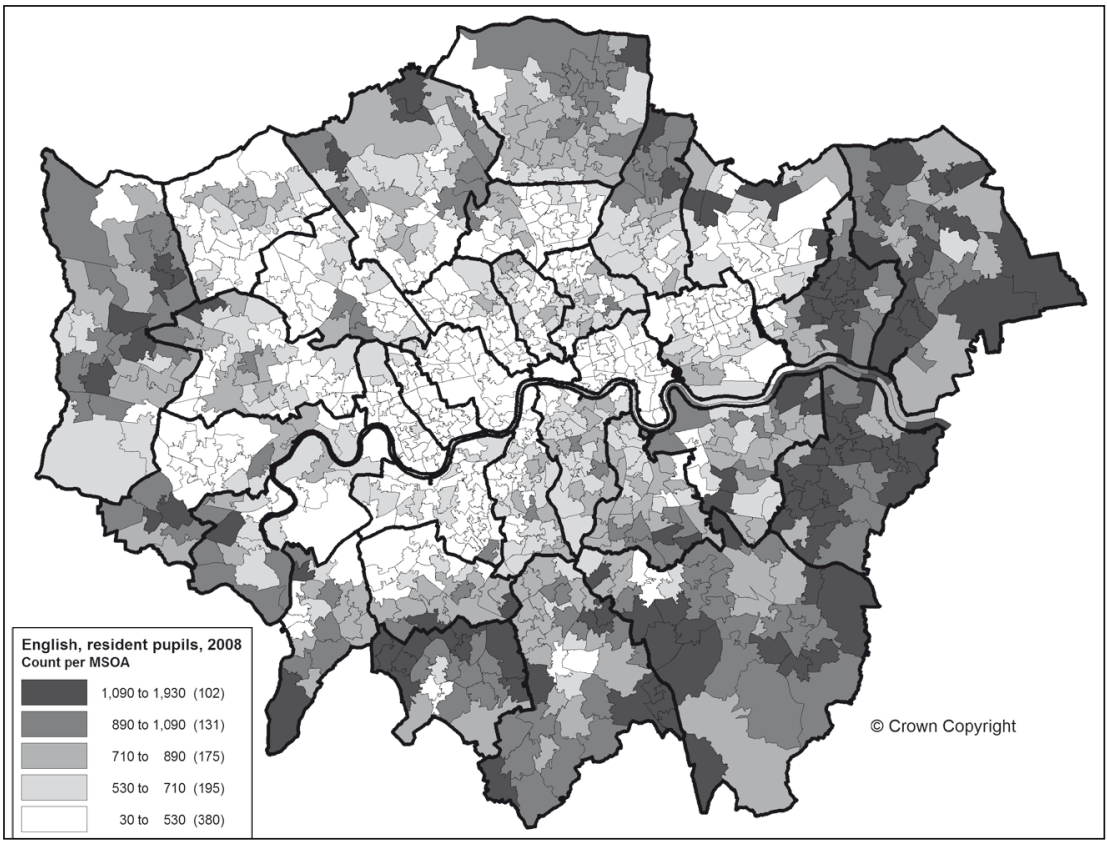

Karte 1: Schüler mit Englisch als Muttersprache in London (gemäß „Language Capital Project“)

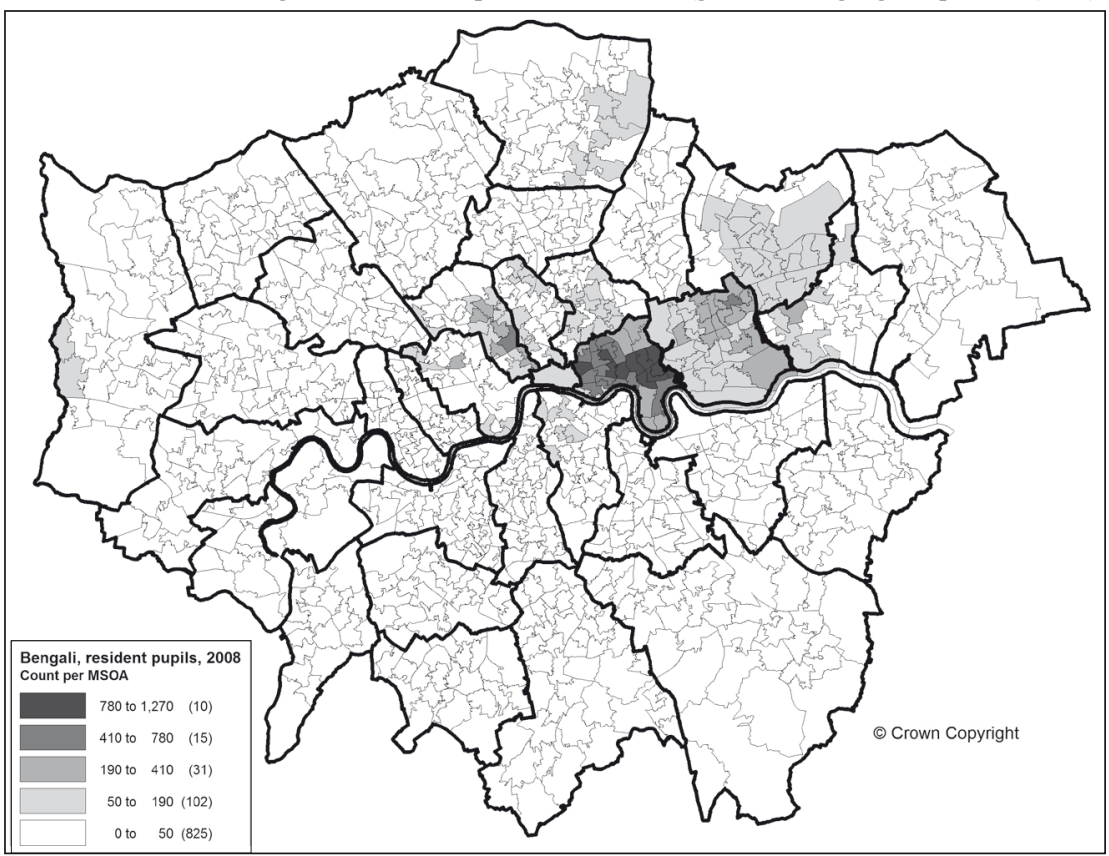

Karte 2: Schüler mit Bengali als Muttersprache in London (gemäß „Language Capital Project“) 


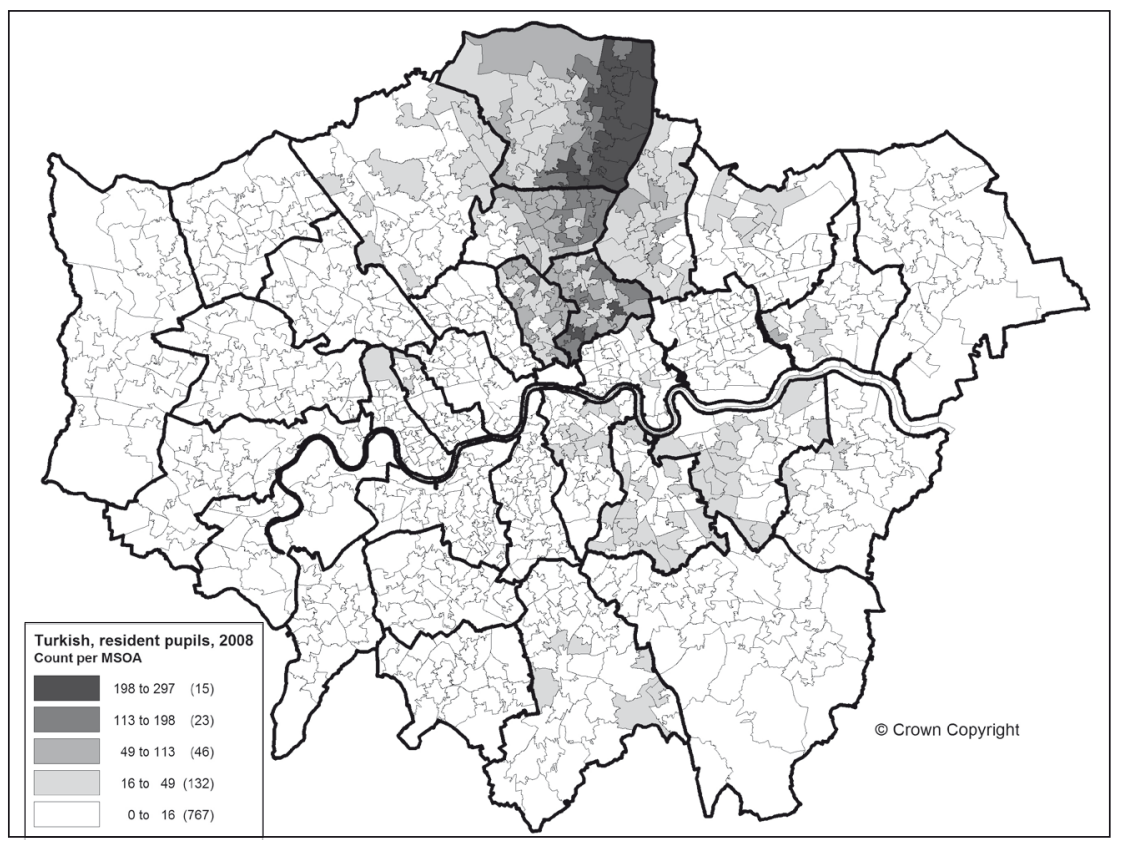

Karte 3: Schüler mit Türkisch als Muttersprache in London (gemäß „Language Capital Project")

Einerseits zeigt zum Beispiel Karte 1, dass Menschen mit Englisch als Muttersprache eher die Randbezirke der Stadt bewohnen, während die Karten 2 und 3 die Tendenz aufzeigen, dass Sprecher anderer Sprachen in bestimmten Bezirken versammelt sind. Indem die in einzelnen Gegenden am weitesten verbreitete Sprache in den Vordergrund gerückt wird, kann diese Vorgehensweise jedoch gleichzeitig dazu führen, dass die jeweilige örtliche Diversität in den überaus globalisierten Nachbarschaften unterrepräsentiert wird. Zudem besteht das Risiko, das Ausmaß der Beständigkeit dieser Ansiedlungen überzubewerten, wo doch die Bevölkerungen zum Teil sehr mobil sind und sich ständig verändern. Eine unbeabsichtigte Konsequenz des Zählens und bildlichen Darstellens von Sprachen ist daher auch die Bestätigung der normativen politischen Diskurse, die auf der Akzeptanz und Glaubwürdigkeit beständiger sozialer Kategorien beruhen. Die heteroglossische Lebenswelt, die jeder in einer großen Metropole bewohnt, steht im Widerspruch zur konsensorientierten Vorstellung von Integration und sozialer Verflechtung sowie zum politischen Druck, Diversität zu kontrollieren und zu regeln.

Wenn man also Menschen auf „Sprecher einer bestimmten Sprache“ reduziert, übersieht man die Chancen und die Grenzen, die ihre eigentlichen Sprachwelten kennzeichnen, und vernachlässigt die Tatsache, dass Umstände und biografische Bedingungen für sie entscheidend sind. Dabei 
übergeht man nicht nur die stilistische Reichhaltigkeit individueller Repertoires sondern auch die disparaten Muster sozialer Beziehungen, die diese Repertoires möglich machen. Individuelle sprachliche Bedürfnisse können einen Wunsch nach Integration und Teilhabe beinhalten, dieser Wunsch kann aber verschiedene Formen annehmen (siehe z.B. Pfaff 2010; Schroeder 2007). Sich in die so genannte Mehrheitsgesellschaft einzugliedern, macht es zweifellos nötig, deutsche Sprachkenntnisse - ob Standardsprache oder Dialekt - zu erlangen, um effektiv mit einsprachigen Deutschsprechern kommunizieren zu können. Sich aber als Berliner durch Teilnahme an kosmopolitischen Gemeinschaften mit informellen und flüchtigen Beziehungen zu integrieren, benötigt normalerweise komplexere Praktiken, die vielsprachige Ressourcen und Deutsch als lingua franca vermischen.

Die Mitglieder eines Boule-Clubs, zum Beispiel, die sich freitagabends am Ufer des Landwehrkanals in Berlin versammeln, kommen aus vielen Bezirken zusammen, um zu spielen. Ein Spiel kann zu jeder Zeit Berliner verschiedener Nationalitäten einschließen - etwa einen Deutschen, einen Italiener, einen Haitianer, einen Argentinier - und neben Deutsch als lingua franca auf dem Boule-Feld werden andere Sprachen für taktische Absprachen zwischen Mitgliedern einer Mannschaft oder zum Wortwechsel mit Zuschauern gebraucht. Zu Spielende zerstreuen sich die Mitglieder wieder, lösen diese örtlich konzentrierte „,community of practice“ wieder auf, und treten jeweils wieder in einen anderen Bereich ihrer Lebenswelt ein (siehe auch Lamarre/Lamarre 2009).

Darüber hinaus gibt es auch andere biografische Aspekte des Sprachprofils eines Migranten, die nicht angemessen von konventionellen Kategorien von Motivation erfasst werden können, die aber für die bisweilen besonderen Strukturen sprachlichen Wissens verantwortlich sind. Betrachten wir folgende Beispiele: ${ }^{2}$

- Für Murat, der einsprachig mit Türkisch aufwuchs, bevor er die Türkei verließ, um in Berlin zu studieren, ist Deutsch nun ein wesentlicher Bestandteil seines Repertoires - für berufliche Zwecke, um mit Schulen zu kommunizieren, für die seine Agentur arbeitet, die pädagogische Projekte gegen Antisemitismus anbietet; mit verschiedenen Regierungsbehörden, die Gelder vermitteln; und auch zu Hause mit seiner deutschsprachigen Frau und zweisprachigen Tochter. Seine Agentur ist jedoch fast komplett mit Menschen besetzt, die einen Migrationshintergrund haben und obwohl sie Deutsch als lingua franca benutzen, schafft die Kenntnis anderer Sprachen ein symbolisches Kapital, das ihre Glaubwürdigkeit in vielsprachigen Klassenzimmern stärkt.

Diese Beispiele entstammen dem Korpus biografischer Interviews mit MigrantInnen in Berlin, das weiter unten besprochen wird. 
- Joseph, der mit Tamilisch und Englisch aufwuchs, war Teil einer Gruppe von Facharbeitern, die in den 1970er Jahren in Indien angeworben wurden, aber obwohl er einen deutschen Sprachkurs in Bangalore absolvieren musste, bevor er nach Deutschland kommen konnte, war kurz nach der Ankunft klar, dass seine elementaren Sprachkenntnisse für die Bedürfnisse an seinem Arbeitsplatz ausreichend waren. Trotzdem eignete er sich nach und nach ein gehobenes Deutsch an, das er noch um Kommunikationsstrategien in Deutsch als lingua franca erweiterte. Dadurch war er in der Lage, sowohl als Ausbilder für andere ausländische Arbeitskräfte zu arbeiten, als auch als Vermittler oder „language broker“ (Sprachmakler: Norton 2000) zwischen ihnen und den Führungskräften (siehe auch Faist 2009, S. 180). Seine „Multikompetenz" (Franceschini 2011) nimmt großen Raum in seiner Lebensgeschichte ein, wo er sich zwischen seinem deutschen Arbeitsplatz einerseits und der tamilischen Kirche andererseits verortet.

- Und bei Hoa, die im Laufe der letzten 60 Jahre dreimal von Vietnam nach Deutschland kam, hatte nicht nur die deutsche Sprache, sondern vor allem ihre Zweisprachigkeit verschiedene Funktionen in verschiedenen Lebensabschnitten. Sie kam zuerst 1955 in die DDR, zusammen mit anderen Kindern, die in der frühen Phase des Vietnamkrieges aus Hanoi evakuiert und auf eine zweiwöchige Reise mit dem Zug via Peking, die Mongolei, Moskau und Warschau geschickt wurden. Nach dem Schulabschluss in der DDR machte sie zunächst eine Ausbildung zur Optikerin, bevor sie nach Vietnam zurückkehrte. In den späten 1960er Jahren wurde sie ausgewählt, um ein Aufbaustudium der Chemie in der DDR zu absolvieren, und ging dann wiederum zurück nach Hanoi, wo sie mehrere Jahre als Übersetzerin und Dolmetscherin in einem Forschungsinstitut arbeitete (sie arbeitete sogar kurzfristig als Übersetzerin für deutsche Journalisten während blutiger Kampfhandlungen in Kambodscha). Zuletzt übersiedelte sie 1987 in die DDR, im Zuge eines großen Zustromes von Vertragsarbeitern (Krüger-Potratz 1991; Weiss/Dennis (Hg.) 2005). Sie kam speziell als Gruppenleiterin, die damit beauftragt war, eine Gruppe von Arbeitern zu koordinieren und zu beaufsichtigen, und mit der Betriebsleitung zu vermitteln; es war nicht vorgesehen, dass diese Arbeiter, die in separaten Wohnbereichen untergebracht wurden, Deutsch lernen sollten, und so (ähnlich wie bei Joseph, nur unter ganz anderen Umständen) stellte ihre Zweisprachigkeit auf praktische Weise sicher, dass diese ausländischen Arbeitskräfte effizient eingesetzt werden konnten.

Der Gebrauch der deutschen Sprache ist daher vielschichtig und oft verbunden mit anderen Sprachen in sehr speziellen sozialen Umständen und für bestimmte Zwecke, die womöglich wenig oder gar nichts mit „Integra- 
tion“ in die sozialen oder kulturellen Traditionen der „Aufnahme-Gesellschaft" zu tun haben. Gleichzeitig hat dieser Gebrauch von Sprache und die Erfahrungen damit (potenziell) entscheidende Auswirkungen, sowohl in Bezug auf die Leben einzelner Menschen als auch in Bezug auf die Gesellschaft, in der sie leben. Die Beiträge von Katharina Brizić und Anne Betten in diesem Band zeigen auf, wie eine enge Beschäftigung mit einzelnen Menschen etwas über das ,geheime Leben der Sprachen“ (Brizić 2006) aufzeigen kann, das sonst immer verborgen bleiben würde. Ich möchte vorschlagen, dass das gewinnbringend mit einer reflektiven, biografischen Herangehensweise verbunden werden könnte, die Erfahrungen mit Sprache erforschen kann, um die „heteroglossische Lebenswelt“ zu verstehen, in der heutige Stadt-Bewohner leben, und sie als „Feld transformierender Handlungen" (siehe oben) zu interpretieren.

\section{Sprachwelten und Sprachbiografien}

Biografische Ansätze wurden in den letzten Jahren in verschiedenen Disziplinen weiterentwickelt: zum Beispiel in der Soziologie, den Gesundheitssowie Erziehungswissenschaften, wie auch in dem fest etablierten Feld der „oral history“. Forschung speziell zu Sprachbiografien wurde aus verschiedenen Perspektiven und anhand verschiedener Formen von Daten durchgeführt, einschließlich sowohl schriftlicher als auch mündlicher Quellen (Pavlenko 2007, Tophinke 2002 und Fix 2010 bieten einen Überblick über dieses Forschungsfeld; siehe auch Burck 2005; Franceschini (Hg.) 2010; Franceschini/Miecznikowski (Hg.) 2004; Lamarre/Lamarre 2009; Kramsch 2009; Meng 2001; Nekvapil 2000, 2003; Norton 2000).

Ich möchte gerne über einige Beispiele aus einer Reihe biografischer Interviews aus dem Jahr 2011 sprechen, die in der ersten Phase eines größeren Projektes zu Sprache, Migration und Raum in Berlin durchgeführt wurden. Die Gesprächsteilnehmer während dieser Projektphase sind alle Einwanderer der ersten Generation, und können daher aus erster Hand über die Erfahrungen mit den Veränderungen der Lebenswelten berichten, die die Migration mit sich bringt. Sie kamen aber alle zu verschiedenen Zeiten nach Deutschland, von den 1950er Jahren bis heute. Sie haben verschiedene ethnische Zugehörigkeiten, kommen aus verschiedenen Ländern und haben verschiedene Migrationslaufbahnen und verschiedene Berufe. Sie rangieren im Alter zwischen 20 und 70 und leben in verschiedenen Bezirken.

Ich möchte mich auf einen Aspekt der Studie beziehen, die von Irina Liebmanns Berliner Mietshaus (2002) und dem Projekt Schillerpromenade (Bezirksamt Neukölln (Hg.) 1996) inspiriert ist und Ingrid Gogolins Aufforderung nachkommt, die Geschichten der Bewohner eines einzelnen Hauses nachzuvollziehen (siehe oben). Ich werde dieses Haus Mareschstraße 74 
nennen; die Straße gibt es wirklich, das Haus nicht. Es ist eine Fiktion, die als öffentliche Fassade für das echte Haus dienen muss, dessen Bewohner an dieser Studie teilnahmen, und das auf seine eigene Weise ein Mikrokosmos der Stadt ist: Seine Bewohner - zur Zeit, als die Studie gemacht wurde - waren Deutsche, Briten, Türken, Polen, Israelis, Franzosen, Thailänder, Russen und Dänen. Das Ziel dieses Teils der Studie ist letztlich, eine Art Biografie des Hauses zu erstellen, aber hier will ich mich auf die Lebensgeschichten von nur zwei Bewohnern beschränken. Beide sind Polen und beide sind etwa im gleichen Alter, haben aber sonst augenscheinlich nichts gemeinsam.

Ich will mich hier darauf konzentrieren, wie die Erzähler ihre Lebensgeschichten um transformierende Ereignisse herum arrangieren, in denen Sprache in irgendeiner Weise eine Rolle spielte. Es geht also darum, zu verstehen, was „biografische Arbeit“ bedeutet, oder was es bedeutet, „Lebensgeschichten biografischer Transformation [zu erstellen] [...] Lebensgeschichten, in denen sich grundlegende Orientierungen ändern" (Treichel/Bethge 2010, S. 113).

,Marek' ist 41 Jahre alt, ein mäßig erfolgreicher Geschäftsmann (Autohändler und Immobilienbesitzer), der erstmals 1977 im Alter von 7 Jahren ins damalige Westberlin kam. Sein Vater war während einer Geschäftsreise zwei Jahre zuvor geflohen und konnte schließlich seine Frau und seinen Sohn zu sich holen. Abgesehen von einer Zeit von etwa 18 Monaten in den 1990er Jahren lebt Marek seit dieser Zeit in Berlin. Seine Lebensgeschichte vollzieht sich jedoch in zwei Migrationszyklen, die jeweils von Brüchen und Krisen und deren Milderungen oder Lösungen geformt und durchsetzt sind. Alle beruhten in irgendeiner Weise auf sprachlich vermittelten Handlungen, die wiederum die Beschaffenheit seiner Lebenswelt veränderten.

Der erste Zyklus - der erste Akt in seiner Inszenierung seiner Lebensgeschichte (siehe Betten 2010, S. 35) - beginnt mit seiner Ankunft in Berlin, die den ersten großen Bruch in seinem Leben darstellt. Damals sprach er nur Polnisch, aber sein Vater besorgte ihm täglichen Nachhilfeunterricht in Deutsch bei einer Nonne, und auch der Vater sprach nur Deutsch mit ihm, um ihm beim Erlernen der Sprache zu helfen. Mareks Mutter lernte nie Deutsch und sprach daher nur Polnisch mit ihm. Er fügte sich gut ein und verließ die Schule im Alter von 16 Jahren, um Polizist zu werden, sein traumberuf. Doch das Ende seiner Ausbildung fiel mit der Wende zusammen, die auch für ihn zum entscheidenden Wendepunkt werden sollte. Es war, wie er sagt, eine heftige zeit: Im Laufe von drei bis vier Jahren starben seine beiden Eltern, er verließ den Polizeidienst, ging wieder zur Schule, um sein Abitur nachzuholen, begann zu studieren und war kurze Zeit verheiratet. Nach einer Zufallsbegegnung mit einem älteren polnischen Ehepaar ging er für eineinhalb Jahre nach Polen, um mit ihnen zu leben, wo er sich mit Gelegenheitsarbeiten wie Übersetzungen und Dolmetscherdiensten über Wasser hielt. 
Der zweite Zyklus beginnt mit seiner zweiten Ehe und seiner Rückkehr nach Berlin. Dort begann er, sich eine Catering-Firma aufzubauen, erlitt dann aber das wichtigste ereignis meines lebens, einen Arbeitsunfall, der sein linkes Bein dauerhaft schädigte und ihn um seine Arbeit brachte. Er und seine Frau bekamen eine Tochter, aber auch diese Ehe endete mit einer Trennung, und er zog sich ganz zurück, weil er sich nicht gleichwertig mit seinen Freunden fühlte, da er keinen Sport mehr machen konnte. Von diesem Zeitpunkt an drehte sich sein Leben eng um ein neues Unternehmen, das er sich aufbaute, und um seine Tochter, die er zusammen mit seiner Ex-Frau aufzieht. Beide Aktivitäten beruhen auf engen, translokalen Beziehungen zwischen Polen und Berlin.

Das Erzählen dieser beiden Migrationszyklen fügt eng das zusammen, was Baynham (2006, S. 188) auf folgende Weise beschreibt: „the intricate relationship between space, time and agency, through which historical time and social space create opportunities, moments where certain kinds of agency become possible." Genau hier werden die transformativen Auswirkungen des Erzählens seiner Lebensgeschichte deutlich: Seine Geschichte besteht aus Fragmenten oder Episoden, die zu einer räumlichen und zeitlichen Logik zusammengefügt werden, die auf wiederholten physischen, sozialen und sprachlichen Grenzüberschreitungen beruht. Marek lebt nicht in einer Art Grenzregion zwischen zwei Gesellschaften: Er zieht vielmehr eine scharfe Trennlinie zwischen den beiden, die den wiederholten Grenzüberschreitungen erst ihre Bedeutung verleiht, und er positioniert sich in seiner Erzählung fest in beiden Gesellschaften, aber auf jeweils verschiedene Art und Weise und beide sind durch persönliche/affektive Beziehungen einerseits und berufliche/geschäftliche Kontakte andererseits verbunden (siehe Auszüge 1 und 2).

\section{Auszug 1: scheidung}

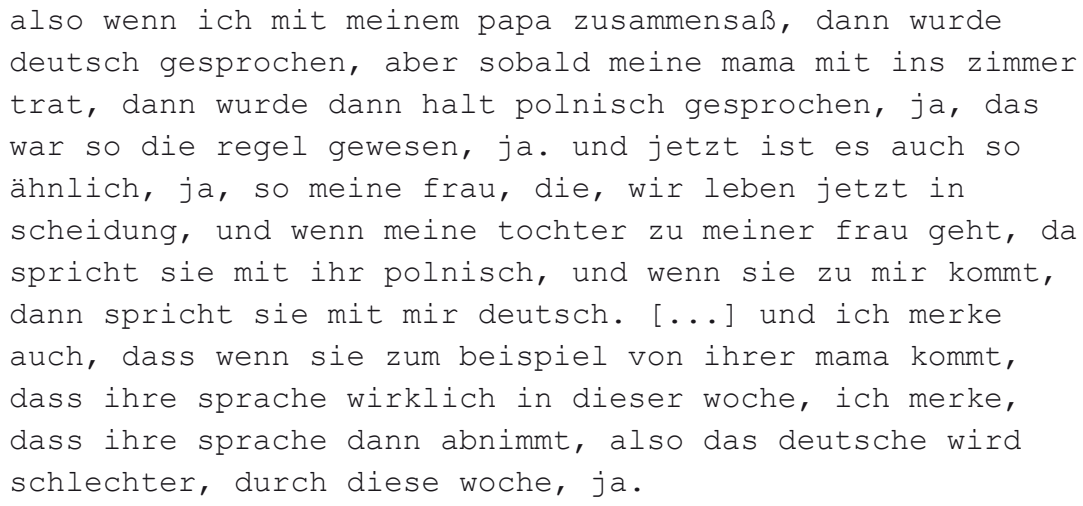




\section{Auszug 2: fließender übergang}

in polen spricht sie polnisch, hier spricht sie deutsch. [...] sie stellt sich da um, wir durchfahren die grenze nach polen und dann sprechen wir auf einmal polnisch mit einander, ja, das ist so fließender übergang, ja, das merken wir manchmal gar nicht, dass wir einmal die sprache wechseln, ja, das war mir sehr wichtig.

Die privaten Sphären in Mareks Lebenswelt sind fast ausschließlich einsprachig (Deutsch in Berlin mit seiner Tochter, Jugendfreunden und Mitbewohnern; Polnisch in Polen mit seiner Tochter, seinen Schwiegereltern und weiteren Sozialkontakten), die öffentlichen sind weitgehend zweisprachig (wo er zwischen deutschen Anbietern und polnischen Käufern vermittelt). Diese symmetrische Anordnung brachte eine komplizierte Laufbahn hervor, in der er sich zwischen zwei Räumen bewegen musste, die mit jedem Wendepunkt in seinem Leben jeweils ihren Charakter veränderten. Das polnische Dorf seiner Kindheit wird zum Idealbild einer harmonischen Welt voller Gemeinschaftssinn und Vertrauen, aus der er abrupt herausgerissen und in eine unbekannte Welt in Berlin versetzt wurde, die eine sprachliche und soziale Grenzerfahrung bedeutete, da er komplett ohne sprache war und sich auf ältere, zweisprachige Kinder verlassen musste, um herauszufinden was sache ist (siehe Auszüge 3 und 4).

\section{Auszug 3: eine heftige zeit - aus polen rausgezogen}

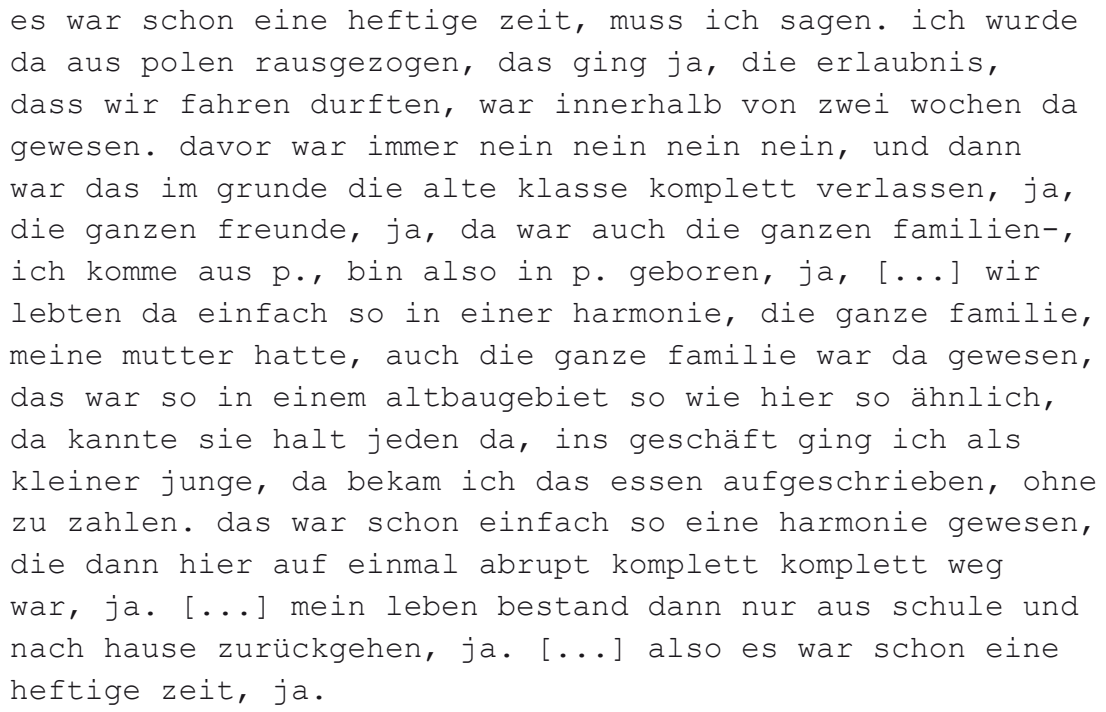




\section{Auszug 4: komplett ohne sprache}

am anfang, ich kann mich erinnern, wie ich da in der klasse saß, also ich hab ja meine ganzen freunde aus der grundschule, da hab ich noch, also zu den besten freunden hab ich noch kontakt, ja, und die erinnern sich immer an die zeit noch, wie ich da in der schule saß und von nix wußte, wie ich ( $\mathrm{xx}$ ) wenn pause war, wußte ich nicht mehr in pause, was ich machen sollte und die erinnern sich immer noch dran, ja. [...] und die erinnerung kommt immer wieder, ja, dass sie sich da erinnern an diese zeit, wie ich da in die klasse kam und und komplett ohne sprache und in den reihen saß im grunde völlig hilflos und da musste jemand von den höheren klassen kommen und mir bescheid sagen, was jetzt sache ist, ja.

Diese fremde und eingeschränkte Welt war zunächst auf die drei Eckpunkte Zuhause, Schule und Kloster begrenzt, aber nach und nach entwickelte sich daraus ein Raum voller Möglichkeiten und Selbsterfüllung, als seine erlernte und fürsorglich geförderte Zweisprachigkeit als soziales Kapital einen Wert erlangte (siehe Auszug 5).

\section{Auszug 5: mit dem megafon sprechen/sprachspange}

damals war 89 die grenze gefallen, und da war hier immer, ach nee 88 war das schon gewesen, da warn hier immer diese märkte. da kamen die polen mit den waren aus polen und haben dann diese im grunde illegalen märkte dann eröffnet, wo sie die sachen verkauft haben, fleisch, irgendwelche handgemachte sachen, und das war halt nicht erlaubt, in so einer großen masse wie es da war, und es wurde wahrscheinlich auch mit zigaretten gehandelt, was verboten war, und alkohol, was verboten war, und dann wurde natürlich immer einen dolmetscher gebraucht, ja, der mit dem, öh, der mit dem mikrof- mit dem megafon da die leute, und dann hab ich da diese rolle übernommen, was für mich auch schwer war, ja, weil das warn ja irgendwie auch meine landsleute, ja, und man musste sie oftmals wegscheuchen oder so, ja. aber ich hab das dann mit dem megafon gemacht und dann hab ich, nach drei monaten hab ich öh ne sprachspange bekommen, dass ich als offizieller dolmetscher bei der polizei arbeiten darf, ja, zwar nix unterschriebenes aber ich durfte öh halt dieses mit dem megafon sprechen, ich durfte die leute öh, auf der 
dienststelle durfte ich dolmetschen, ja, und da war ich ganz stolz, weil da war ich, glaub ich, achtzehn jahre oder so, und auf einmal war ich mit dem chef da auf dem wagen gewesen. das [die zweisprachigkeit] war ein vorteil für mich damals, ja [...] und auch die kenntnisse, das war ein vorteil für mich, dass ich die hatte, auch in der perfektion durch meine mutter halt, dass ich das nicht verlernt habe.

Diese Möglichkeiten waren jedoch auch mit Kosten verbunden. Sowohl er als auch seine Mutter mussten ihre polnische Staatsbürgerschaft aufgeben, damit er überhaupt in den Polizeidienst eintreten konnte, und seine erste Gelegenheit, sein zweisprachiges Repertoire anzuwenden, machte es nötig, dass er wortwörtlich als Stimme des deutschen Staates kurz vor und während der Wende den illegalen grenzübergreifenden polnischen Handel regeln musste. Die Händler, die er wegscheuchen musste, sind als Andere dargestellt (polen), zu denen er eine ambivalente Beziehung hat (sie sind irgendwie meine landsleute), und seine Kommunikation mit ihnen geschieht nur in eine Richtung, in dem er Befehle durch das Megafon, das offizielle Dienstinstrument, ruft. Diese Konfrontation ist auch eine ironische Vorwegnahme seiner eigenen späteren grenzüberschreitenden Aktivitäten.

Der Tod seiner beiden Eltern in rascher Folge direkt nach der Wende ist der erste von zwei Unglücksfällen, die sein Leben bestimmten. Dieser ist gefolgt von einer Reihe von Krisen, die auf eine kurze Passage in seiner Lebensgeschichte konzentriert ist, bevor er sich wieder ausgreifender seiner ersten Rückkehr nach Polen mit Mitte 20 widmet. Zum Land seines Ursprungs hat er Zugang dank seiner Polnischkenntnisse, die seine Mutter ihm so gewissenhaft erhalten half, und zu diesem Zeitpunkt wird Polen sein Rückzugsort, der Sicherheit und Wärme in einer Familie bedeutet (siehe Auszug 6).

\section{Auszug 6: heimat/ein ganz anderes nebenherleben}

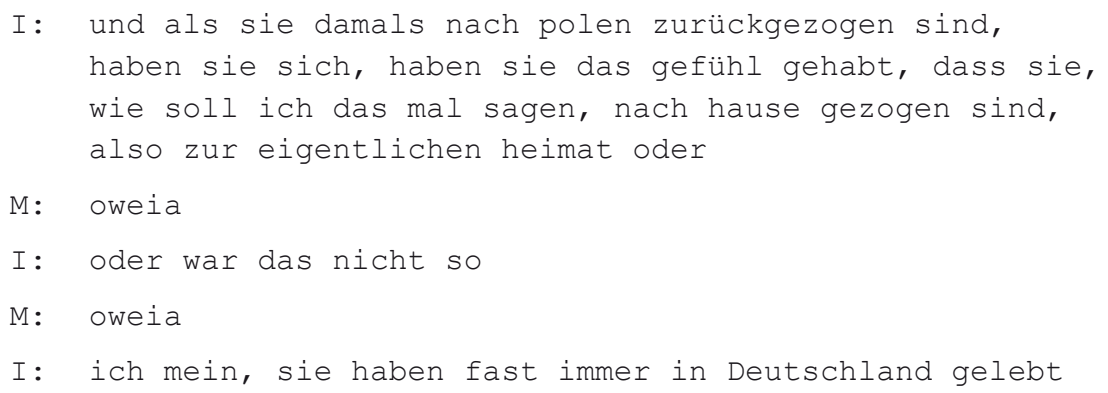


M: also es ist ja jetzt immer noch so, dass ich, wenn ich nach polen fahre, immer noch so' $n$, also es wechselt wirklich, ich hab da wirklich so ne andere empfindung, wenn ich denn da bin, ja, also mein verhalten ändert sich auch ein bisschen, ich merk das selber, ja, also wenn ich mit den leuten spreche und so, ja, es ist halt, aber es kommt auch dadurch, dass ich da halt mehrere menschen um mich habe, ja, hier bin ich eher so 'n einzelgänger, ja, und dort ist halt, da kommt der, und da kommt der

I: mehr geselischaft

M: mehr gesellschaft, der begrüßt mich, der sieht mich, der hält mich an, komm mal mit mir, und es ist halt ein ganz anderes nebenherleben, ja, weil ich die leute, das ist ein kleines dorf, wo, so 'ne stadt, viertausend leute, ja, der papa [sein schwiegervater], der opa, ist kulturdirektor, also man kennt mich, jeder kennt mein gesicht da im grunde, ja, und es ist halt so, dass wenn ich dann da bin, dann ist halt ein ganz anderes gefühl für mich als hier, ja, weil da einfach jeder will mit dir sprechen, jeder hat irgendwie was zu sagen. ich hab ja auch die wohnung ein bisschen renoviert, da hab ich den arbeiter aus polen, aus $x$, also wo meine frau herkommt, genommen, dann hab ich mal den arbeiter hergenommen, denen hab ich was erledigt, ich bin so 'n typ, ich kann nicht nein sagen und öh hab so viele gefälligkeiten den leuten getan, dass sie jeder mich in positiver erinnerung hat, ja, und vielleicht verstärkt das dann dieses heimatgefühl mehr, als es in wirklichkeit ist, ja.

I: $\quad$ so, $\mathrm{mhm}, \mathrm{mhm}$

M: aber als ich damals nach polen gezogen bin, war halt schön gewesen, war ich dann auch, waren meine eltern tot gewesen, hier ist meine beziehung in die brüche gegangen, zu meinem sohn hab ich gar keinen kontakt mehr gehabt, und dort, bei dem freund, der hat eine mutter, der hat einen vater gehabt, öh, und die haben mich da eingegliedert wie ihren eigenen sohn, ja. wir saßen zusammen am mittagstisch, haben gegessen, wir saßen am frühstücks-, haben zusammen gefrühstückt, 
und es war halt so, da hab ich mich schon wie zu heher wie zu hause gefühlt, vielleicht nicht wie in pol-wie in der heimat, aber wie zu hause gefühlt, ja, na wahrscheinlich auch ein bisschen wie in der heimat, aber es war halt dieses, dass man zusammen am frühstückstisch saß, zusammen am mittagstisch saß, ja, es wurde für einen gekocht, es war halt ein anderes leben als hier in in

I: ein schönes familiengefühl

M: genau genau genau. die polen sind halt sehr gastfreundich. obwohl ich ein fremder war, haben die mich da mit offenen armen angenommen und ich hab da ein jahr wirklich dieses leben genossen, ja, mit dem sohn von ihnen, ja, ist wahrscheinlich auch ein bisschen heimat, das stimmt.

Der zweite (Aus-)Wanderungsakt beginnt mit seiner Rückkehr nach Berlin, zusammen mit seiner zweiten Frau, mit der er später seine Tochter Ania haben wird. Berlin wird wiederentdeckt als Ort der Möglichkeiten, als er sich eine neue Karriere als Geschäftsmann aufbaut und durch seinen Sport lebendige soziale Kontakte entwickelt. Diese beiden Bereiche werden jedoch durch den zweiten Unglücksfall beschnitten, den Unfall. Damit wird Berlin plötzlich wieder zum eng begrenzten Raum in seiner Lebenswelt, und das Öffentliche und das Private treffen in dem Haus zusammen, das ihm zum Teil selbst gehört (er ist Eigentümer mehrerer Wohnungen), wo er zum Beirat gehört, und seine Wohnung ist sowohl sein Büro als auch das Zuhause seiner Tochter (siehe Auszug 7).

\section{Auszug 7: ich kann das nicht mitmachen}

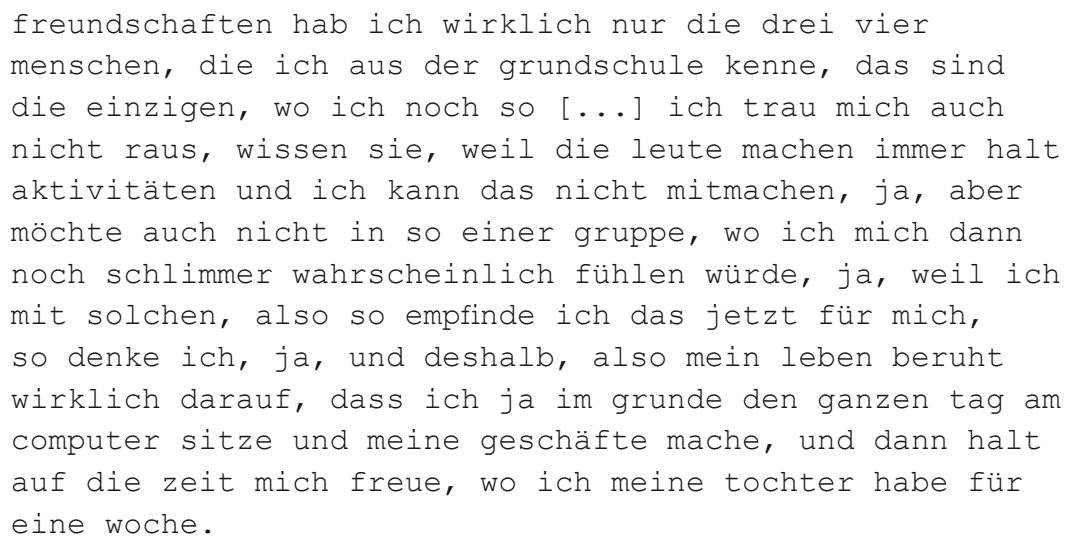


Jetzt hat er jedoch private und weitere soziale Kontakte nach Polen, die einen Raum voller gegenseitiger Abhängigkeit schaffen. Diese Kontakte beruhen auf den hochentwickelten sprachlichen Ressourcen, auf die er zurückgreifen kann, wie auch auf seiner persönlichen Sprach(en)politik: Er wiederholt nun mit seiner Tochter (die jetzt im gleichen Alter ist wie er, als er zuerst nach Berlin kam) den gleichen Prozess der zweisprachigen Entwicklung, die er durchmachte (siehe nochmals Auszüge 1 und 2). Berlin ist Anias multikulturelles und vielsprachiges Zuhause (ihre Schulkameraden sind Russen, Araber, Argentinier und Italiener) und Mareks geschäftliche Niederlassung. Die kleine Stadt in Polen, wo Anias einsprachige Großeltern leben und Mareks Geschäftspartner niedergelassen ist, ist zugleich auch ein lebendiger, geselliger sozialer Raum für Marek: Sowohl seine private Beziehung zu einer bekannten Person im Ort (seinem Schwiegervater) und seine geschäftlichen Aktivitäten, die örtlichen Arbeitskräften berufliche Perspektiven in Berlin eröffnen, schaffen auch ihm Zugang zu sozialen Engagements, die ihm in Berlin verschlossen sind (siehe nochmals Auszug 6).

Diese Entwicklung und der Einsatz seines sprachlichen Repertoires bestimmen und spiegeln den Rhythmus von Mareks Leben, und verweisen in bestimmten, einschneidenden Momenten in seinem Leben auf die Ausprägung einer komplexen Subjektivität. Indem er seine Geschichte erzählt, erschafft sich Marek selbst als Figur, die in der Zeit aktiv handelt: Er nimmt Teil an wiederholten Wanderungsakten über die deutsch-polnische Grenze hinweg, aber unter jeweils radikal unterschiedlichen geschichtlichen Bedingungen; er hat aktiven Teil an der Kontrolle dieser Grenze während der Wende, und beteiligt sich schließlich in sowohl persönlicher als auch beruflicher Eigenschaft an transnationalen/translokalen Aktivitäten über diese Grenze hinweg.

„Beata' ist etwa im gleichen Alter wie Marek, aber sie kam zuerst 1991 nach Berlin. Sie konstruiert ihr Leben als eine Geschichte der Entfremdung, wo Ort und geografischer Umzug vor allem für die (Neu-)Aushandlung ihrer persönlichen Beziehungen von Bedeutung sind. Ihre Lebensgeschichte unterscheidet sich von Mareks in beinahe jeder Hinsicht. Auch sie stellt ihren Umzug nach Berlin allerdings als „Flucht" dar, aber in ihrem Fall ist es keine Reise mit ihrer Mutter, um eine Familie wieder aufzubauen, sondern eine Flucht vor ihrer Mutter - ich bin auch geflüchtet, von meiner familie, von meiner mama-und vor ihrer Muttersprache. Und auch Beata entwickelt eine engere Beziehung zur deutschen Sprache als zur polnischen, aber sie geht noch weiter als Marek und bezeichnet Deutsch als Zuflucht und neues Zuhause (siehe Auszug 8). 


\section{Auszug 8: die polnische sprache ist eine herausforderung}

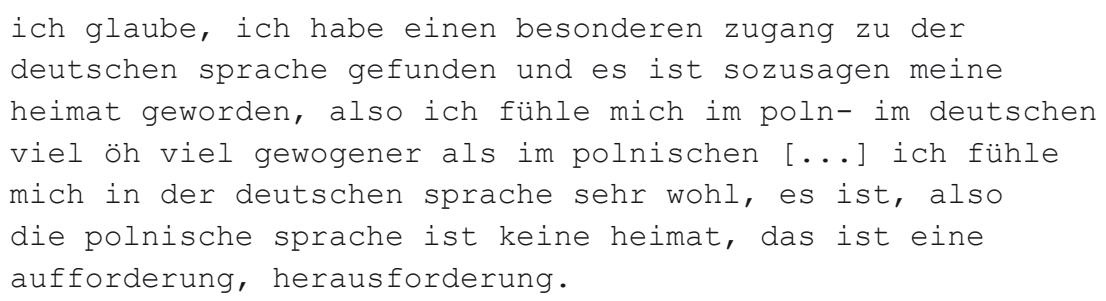

Für sie ist der Umzug tatsächlich ein scheinbar endgültiger Akt, und obwohl ihr wachsendes sprachliches Repertoire es ihr auch erlaubt, persönliche und berufliche Verbindungen zum Land ihres Ursprungs (wieder-)herzustellen, scheinen Polen und Polnisch ein entferntes und fremdes Gebiet zu bleiben. Durch ihr Studium der Kunstgeschichte in Berlin entdeckte sie ihre Berufung zur zeitgenössischen Kunst und ihre erste Arbeit in einem Institut für Kulturaustausch brachte unerwartete Begegnungen mit der Sprache, die ihr inzwischen fremd geworden war (siehe Auszug 9).

\section{Auszug 9: konfrontation mit der sprache}

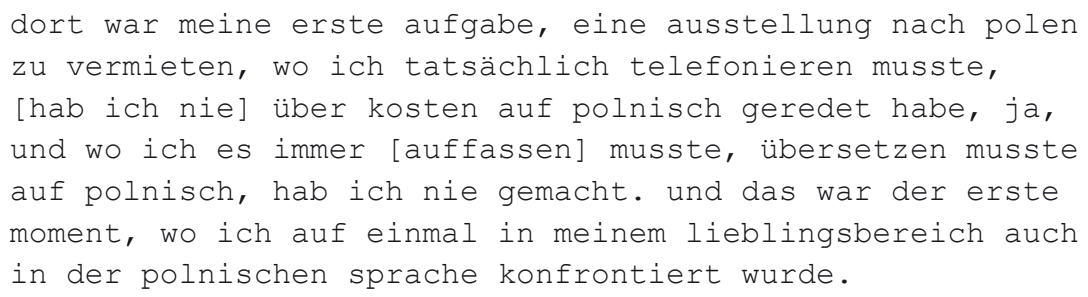

Beata beginnt und beendet ihre Geschichte mit Erzählungen zu sprachlichem Versagen, die jeweils, direkt oder indirekt, mit der problematischen Beziehung zu ihrer Mutter zu tun haben. Neben der Flucht vor ihrer Mutter beruhte ihr Umzug nach Berlin, so sagt sie, auf dem Vorsatz, ihre ungenügenden Leistungen in Deutsch, die ihr einen schlechten Schulabschluss beschert haben, aufzuholen. 20 Jahre Studium und Arbeit in einem deutschsprachigen Umfeld haben es ihr ermöglicht, fließend Deutsch zu lernen, in dem sie sich selbstbewusst ausdrücken kann. Aber obwohl sie dadurch eine Meta-Sprache erlangt hat, in der sie ihre frühen Erlebnisse auf Deutsch artikulieren kann, bleibt sie doch mit ihrer Mutter auf einem Gebiet gefangen, das ihnen beiden fremd ist, wenn sie versucht, sich ihr emotional auf Polnisch zu nähern (siehe Auszug 10).

\section{Auszug 10: gefühle übersetzen}

B: also auch zum beispiel bei dieser versöhnung mit meiner mutter ist eigentlich absurd. sie, wir haben, 
sie hat mich mal besucht und wir haben da einen

konflikt gehabt, ja

I: sie hat dich hier besucht?

B: ja, sehr oft, aber einmal als sie mich besucht hat, da gabs eine konfliktsituation, ja, und sie kann überhaupt nicht reden, also sie [weinte] und ging weg, ja, und öh und ich kann das nicht mehr aushalten, das ist auch als sie, sie ist mit mir umgegangen als wie ich klein war, aber das geht jetzt nicht mehr. dann hole ich sie zurück sozusagen und versuche [sie zu trösten] und ich arbeite dann mit einem wörterbuch [I: mhm], weißt du, weil ich, weil ich alles, was ich sagen will, über die gefühle, aus dem deutschen übersetzen muss.

Die emotionale Distanz zwischen Beata und ihrer Mutter scheint sich in all der Zeit nicht verringert zu haben. Was sich aber verändert hat - und diese biografische Verschiebung sieht man in Auszug 10 am Wechsel von der Vergangenheits- zur Gegenwartsform - ist, dass Beata sich jetzt als aktive Figur in ihrer Geschichte positioniert (im Sinne Goffmans 1981), die passive Haltung des Kindes aufgibt (siehe Auszug 11) und eher eine elterliche Haltung einnimmt, indem sie kontrolliert und tröstet; auch wenn ihr noch immer das sprachliche Vermögen fehlt, ihre Gefühle frei auf Polnisch auszudrücken, besitzt sie nun die intersprachliche Fähigkeit, sich zwischen zwei Sprachen zu bewegen, und auch genug Erfahrung damit, um die Situation meistern zu können.

\section{Auszug 11: ich hab mich nicht getraut zu sprechen}

B: ja, meine mutter stand sozusagen da mit so ganz kräftigen muskeln [I: ja?], die hat auch gewalt angewendet und so, also schon ich hab mich einfach nicht getraut zu sprechen, meine meine wünsche zu äußern und öhm am am am meistens hab ich gewonnen, wenn ich gar nichts gesagt habe, wenn ich ganz ruhig, still war.

I: so, mhm, du hast dich zurückgezogen

B: genau, aber das hängt tatsächlich damit zusammen, dass ich die polnische sprache öh nie so öh so unbefangen gelernt habe [I: $\mathrm{mhm}$ ] sondern die polnische sprache war immer eher mit der schule verbunden, wo man schreiben musste, wo man auftritte halten musste [I: mhm], obwohl ich geliebt habe zu lesen, ich hab alles nur aufgenommen. 
Zwischen diesen beiden Konfliktpunkten entwickelt Beata eine Erzählung, in der ihre Erfahrung mit Sprache jeweils eng verknüpft ist mit emotionaler und intellektueller Einschränkung einerseits und Befreiung andererseits. Orte sind eine relevante Kategorie in diesem erzählerischen Prozess, insbesondere als Räume, in denen besondere sprachliche Ressourcen persönliche Beziehungen und Entwicklungen entweder behindern oder befördern. Polen ist der problematische Familien-Raum, wo sowohl sie als auch ihre Mutter in einem sprachlich verarmten Umfeld aufwuchsen (siehe Auszug 12), und in ihrem polnischen Leben stellen Männer Figuren dar, die sich entweder nicht artikulieren können, schweigen, oder sie überhaupt verlassen; die handelnden Figuren sind die Frauen. Die Familie ist, so sagt sie, männerberaubt (siehe Auszug 13) und matriarchal geregelt.

\section{Auszug 12: sprachstörungen}

B: also ich öhm es ist mir angenehmer, mich auf deutsch zu unterhalten als auf polnisch

I: angenehmer?

B: weil das nicht so anstrengend ist, ich meine, das polnische ist immer [?] stress [?] über die ich mich gedanken gemacht habe, aber es kann auch sein, dass es damit zusammenhängt, weil als ich polen verlassen habe ich auch ein öh öh sprachproblem hatte, ja, ich hatte öhm, oder nicht sprachproblem sondern es war so mit meinem bewusstsein verbunden, dass ich mich nicht zu artikulieren wusste, ich wusste nicht, meine ideen zu äußern, ich wollte die ideen alle, die ideen öhm so geballt aus mir herausbringen, weil ich eben zuhause zum beispiel nie [lernen] nie sprechen so richtig durfte, ja [...] also meine mutter kann auch nicht reden, also in ihrem verhältnis mit ihrer mama war wieder mal so was, dass die, ihre mutter, hat sich im mittelpunkt gestellt und hat niemanden um sich [?], sondern alles alleine [?] und immer um sich geredet [...] sie hatte drei kinder und alle kinder leiden unter sprachstörungen. so der eine, der jüngste onkel ist öh hat, stottert, sehr, ja. öh meine mutter, die sagt kaum was, also wenn sie was vermittelt, wenn sie sich durchsetzen möchte, dann ist das meistens durch schreien [...] und der älteste bruder meiner mutter, das ist öhm, also bestimmt, weiß ich nicht, er wurde, er kann sich nicht ausdrücken [I: mhm], er sucht immer nach wörtern, dann dann gibt er den [?] auf, weil ihm die wörter fehlen. 


\section{Auszug 13: männerberaubt}

meine familie war irgendwie, so, öhm, männerberaubt, also verdammt zu zu dieser beraubung von männern, oder vom schicksal wurde sie beraubt. also meine oma hat ihren mann im zweit- nach dem zweiten weltkrieg verloren, also nicht [?] nach aber ziemlich früh ist er gestorben, da war sie die ganze zeit alleine und hat die kinder großgezogen ohne den vater. [I: mhm] und dann meine mama hat auch, so mein vater hat sich auch mit meiner mutter nicht öh hat sie auch verlassen, ja, und dann hatte sie zu einen anderen geheiratet, aber der kommt auch öh also fünfzehn jahre glaube ich waren sie zusammen, dann hat er sie auch verlassen, und in unserem verhältnis, also meine mutter und ich verhältnis, er hatte öhm eigentlich keine keine stimme, also ich war nicht sein kind, er hatte nichts zu bestimmen über mein schicksal, damals.

Ihre Berliner Lebenswelt ist dagegen eine Art emotionales Exil, wo Beata die Möglichkeit bekommt, indem sie die Fremdsprache Deutsch lernt, sowohl eine neue Stimme zu finden und sich eine persönliche Autonomie zu erarbeiten, zuerst als Studentin und dann als unabhängige Kuratorin von Kunstausstellungen. Die Entwicklung emotionaler Reife geht dabei Hand in Hand mit der Entwicklung emotionaler Ausdrucksfähigkeit, und was zuerst ein Rückzugsraum ist, wird im Verlauf der Erzählung ein Raum der Entdeckung und Möglichkeiten. Das Vakuum, das die fehlenden Männer in ihrer polnischen Welt hinterlassen haben, ist hier nicht mit Männern, sondern mit einer Auseinandersetzung mit kreativen und intellektuell aktiven polnischen Frauen ausgefüllt, die ihre eigenen Erfahrungen als künstlerische Außenseiterinnen in Deutschland mit ihrer bildlichen Vorstellungskraft aufarbeiten (siehe Auszug 14).

\section{Auszug 14: von frau zu frau}

also wir arbeiten zum beispiel jetzt an einer ausstellung [...] und das heißt von frau zu frau, und das, es werden sechs frauen insgesamt, sechs künstlerinnen, teilnehmen, die in unterschiedlichen öhm öhm bereichen der kunst tätig sind. wir haben malerinnen, wir haben fotografinnen, wir haben öh bildhauerinnen, öhm performerinnen, ja [I: mhm], sechs also, drei kommen aus polen, und drei sind polinnen aber sie leben in Deutschland [I: mhm] und dann wollen wir sie zusammenstellen und gucken, mit welchen themen sie sich beschäftigen und öhm und weil sie [?] ausdrücken, wie wichtig ist, was das ist, frau sein in der kunst 
[...] polinnen, die in deutschland leben und wie sie das angenommen haben, was ihnen hier angeboten wurde und wie sie bei sich geblieben sind.

So kann Beata ihre eigene, genderbedingte Erfahrung von Andersartigkeit - in Polen und in Berlin - hinterfragen, indem sie sie in eine künstlerische Erkundung verwandelt: Die Themen der Ausstellungen, die sie zusammenstellt, sind die zentralen Themen ihrer eigenen Lebensgeschichte. Zugleich schafft sie eine bestimmte soziale Kategorie - die der Berliner-polnischen Kunst-Kuratorin - in einem bestimmten kulturellen Umfeld, in das sie sich erzählerisch einbringen kann.

Sowohl Marek als auch Beata haben Migration von Polen nach Berlin als Flucht erfahren, wenn auch unter verschiedenen historischen Umständen und aus verschiedenen Motiven heraus. Ihre Lebenswelten sind jeweils heteroglossisch, nicht nur lokal in Berlin, sondern auch translokal, durch die verschiedenen Wege, auf denen sie verschiedene Beziehungen, persönliche oder berufliche, erhalten oder wiederbeleben. Beide schaffen Darstellungen ihrer Migrationserfahrungen, die die transformativen Auswirkungen sprachlichen Handelns aufzeigen, jedoch jeweils auf eine verschiedene Art und Weise. Und beide nutzen ihre Erzählungen, um ein Selbst zu schaffen, das sich auf sprachlich vermittelte Erfahrungen gründet. Die Geschichten anderer Bewohner der Mareschstraße 74 geben vielfältige Zeugnisse dieser Diversität in Bezug auf sprachliche Repertoires, Ethnizität, Beschäftigung, religiöse Bräuche oder Altersprofile unter einem Dach. Indem man sich jedoch auf zwei Menschen konzentriert, die gleichen Alters, der gleichen Ethnizität und des gleichen sprachlichen Repertoires sind, wenn auch mit äußerst unterschiedlichen Migrations-Laufbahnen, ist es möglich, sich einem bestimmten Aspekt der Diversität zu widmen, der sich in der biografischen Besonderheit ihrer Erfahrungen mit Sprache zeigt.

\section{Schlussfolgerungen}

Die Parameter eines Forschungsfeldes, das wir eventuell als „Soziolinguistik der Superdiversität" bezeichnen mögen, werden in einer wachsenden Anzahl von Publikationen abgesteckt. Als Feld der wissenschaftlichen Auseinandersetzung ist es selbst noch im Prozess der Diversifikation begriffen und es wird in den kommenden Jahren zweifellos noch um weitere Dimensionen erweitert werden. In ihrer Aufarbeitung der Möglichkeiten dieses Forschungsfeldes betonen Blommaert/Rampton (2011) die Ethnografie der Sprache als die vorrangige Vorgehensweise, und handlungsbezogene und semiotische Prozesse und Handlungen verschiedenster Art als die Hauptobjekte der Untersuchung. In diesem Beitrag wollte ich darlegen, dass komplexe Phänomene einer multidimensionalen Analyse bedürfen. Insbesondere 
wollte ich die „,heteroglossische Lebenswelt“ der heutigen städtischen Gesellschaft in Deutschland durch beide Enden des Teleskops betrachten, um zu sehen, welche Art Fragen wir stellen sollten, um Ingrid Gogolins Aufforderung (siehe oben) nachzukommen, eine bessere Wahrnehmung der Textur vielsprachiger Gesellschaften zu erlangen.

Einerseits möchte ich deshalb argumentieren, dass wir tiefergehendere und differenziertere demografische Daten zur sozialen und geografischen Verteilung sprachlicher Ressourcen benötigen. Diese Darstellung müsste sich auf ein historisches Verständnis von Migration und Mehrsprachigkeit gründen und die dynamische und komplexe Beschaffenheit sprachlicher Konstellationen sowie auch die Notwendigkeit, politische Diskurse zu beeinflussen, anerkennen. Andererseits, wenn wir mehr darüber herausfinden wollen, was „Sprache im Leben von Menschen bewirkt" (Blommaert 2003, S. 608), schlage ich vor, dass wir die Forschungen zur interaktionalen mehrsprachigen Praxis um reflexive, biografische Vorgehensweisen erweitern, die sich mehr auf individuelles Spracherleben konzentrieren. Dies stünde in der Tradition der narrativen Analyse, in der der Mensch, der die Sprache verwendet, nicht nur ein Produzent oder Konsument von Texten im Hier und Jetzt ist, sondern auch eine zeitgebundene, handelnde Figur in einer Vielzahl sozialer Prozesse und Handlungen über die Zeit hinweg.

\section{Literatur}

Androutsopoulos, Jannis (2007): Ethnolekte in der Mediengesellschaft: Stilisierung und Sprachideologie in Performance, Fiktion und Metasprachdiskurs. In: Fandrych, Christian/Salverda, Reinier (Hg.): Standard, Variation und Sprachwandel in germanischen Sprachen. (= Studien zur Deutschen Sprache 41). Tübingen, S. 113-155.

Auer, Peter (in diesem Band): Ethnische Marker im Deutschen zwischen Varietät und Stil.

Barni, Monica/Extra, Guus (Hg.) (2008): Mapping linguistic diversity in multicultural contexts. (= Contribuions to the Sociology of Language 94). Berlin/New York.

Baynham, Mike (2006): Narratives in space and time: beyond „backdrop“ accounts of narrative orientation. In: Atkinson, Paul/Delamont, Sara (Hg.): Narrative methods. Bd. 2: Narrative applications. London u.a., S. 176-196. [Ursprünglich in: Narrative Inquiry 13/2, 2003, S. 347-366].

Betten, Anne (2010): Sprachbiographien der 2. Generation deutschsprachiger Emigranten in Israel. In: Zeitschrift für Literaturwissenschaft und Linguistik 160, S. $29-57$.

Bezirksamt Neukölln (Hg.) (1996): Schillerpromenade 27, 12049 Berlin: zum Wandel der Großstadtkultur am Beispiel eines Berliner Mietshauses (Ein Haus in Europa). Opladen.

Block, David (2006): Multilingual identities in a global city. Basingstoke. 
Blommaert, Jan (2003): Commentary: A sociolinguistics of globalization. In: Journal of Sociolinguistics 7, S. 607-623.

Blommaert, Jan (2008): Language, asylum and the national order. (= Working Papers in Urban Languages and Literacies 50). London.

Blommaert, Jan (2010): The Sociolinguistics of globalization. Cambridge.

Blommaert, Jan/Backus, Ad (2011): Repertoires revisited: „knowing languages“ in superdiversity. (= Working Papers in Urban Language and Literacies 67). London.

Blommaert, Jan/Rampton, Ben (2011): Language and superdiversity: A position paper. (= Working Papers in Urban Language and Literacies 70). London.

Brizić, Katharina (2006): The secret life of languages. Origin-specific differences in L1/L2 acquisition by immigrant children. In: International Journal of Applied Linguistics 16, S. 339-362.

Brizić, Katharina/Lo Hufnagl, Claudia (2011): „Multilingual Cities“ Wien. Bericht zur Sprachenerhebung in den 3. und 4. Volksschulklassen. Wien. Internet: http://www. oeaw.ac.at/dinamlex/Multilingual-Cities_Wien-2009_Endbericht-V1Stand20111111.pdf (Stand: 14.05.2012).

Brizić, Katharina/Yağmur, Kutlay (2008): Mapping linguistic diversity in an emigration and immigration context: Case studies on Turkey and Austria. In: Barni, Monica/ Extra, Guus (Hg.): Mapping linguistic diversity in multicultural contexts. (= Contributions to the Sociology of Language 94). Berlin/New York, S. 245-264.

Burck, Charlotte (2005): Multilingual Living: explorations of language and subjectivity. Basingstoke.

Busch, Brigitta (2010): Die Macht präbabylonischer Phantasien. Ressourcenorientiertes sprachbiographisches Arbeiten. In: Zeitschrift für Literaturwissenschaft und Linguistik 160, S. $58-82$.

Cheshire, Jenny et al. (2011): Contact, the feature pool and the speech community: the emergence of multicultural London English. In: Journal of Sociolinguistics 15, S. 151-196.

Chlosta, Christoph/Ostermann, Torsten (2005): „Warum fragt man nach der Herkunft, wenn man die Sprache meint?" Ein Plädoyer für eine Aufnahme sprachbezogener Fragen in demographische Untersuchungen. In: Arbeitsstelle Interkulturelle Konflikte und gesellschaftliche Integration (Hg.): Bildungsdaten und Migrationshintergrund. Berlin, S. 62-74.

Chlosta, Christoph/Ostermann, Torsten (2006): Zur Gestaltung und Begleitung einer fragebogenunterstützten Erhebung bei Grundschulkindern. In: Ahrenholz, Bernt/ Apeltauer, Ernst (Hg.): Zweitspracherwerb und curriculare Dimensionen. (= Forum Sprachlehrforschung 6). Tübingen, S. 55-72.

Collins, James/Slembrouck, Stef/Baynham, Mike (Hg.) (2009): Globalization and language in contact: scale, migration and communicative practices. London.

Decker, Yvonne/Schnitzer, Katja (2011): FreiSprachen. Eine flächendeckende Erhebung zur Mehrsprachigkeit an Freiburger Grundschulen. Internet: www.freidaz.de (Stand: 30.05.2012). 
Deppermann, Arnulf (2007): Playing with the voice of the other: stylized Kanaksprak in conversations among German adolescents. In: Auer, Peter (Hg.): Style and social identities. (= Language, Power and Social Process 18). Berlin, S. 325-360.

Extra, Guus/Gorter, Durk (Hg.) (2001): The other languages of Europe: demographic, sociolinguistic and educational perspectives. (= Multilingual matters 118). Clevedon.

Extra, Guus/Yağmur, Kutlay (Hg.) (2004): Urban multilingualism in Europe: immigrant minority languages at home and school. (= Multilingual Matters 130). Clevedon.

Eversley, John et al. (2010): Language capital: mapping the languages of London's schoolchildren. London.

Faist, Thomas (2009): Diversity - a new mode of incorporation? In: Ethnic and Racial Studies 32, S. 171-190.

Fix, Ulla (2010): Sprachbiographien als Zeugnisse von Sprachgebrauch und Sprachgebrauchsgeschichte. In: Zeitschrift für Literaturwissenschaft und Linguistik 160, S. 10-28.

Franceschini, Rita (Hg.) (2010): Sprache und Biographie. Sonderausgabe. Zeitschrift für Literaturwissenschaft und Linguistik 160.

Franceschini, Rita (2011): Multilingualism and multicompetence: a conceptual view. In: The Modern Language Journal 95, S. 344-355.

Franceschini, Rita/Miecznikowski, Johanna (Hg.) (2004): Leben mit mehreren Sprachen: Sprachbiographien. (= Transversales 9). Bern.

Freywald, Ulrike et al. (2011): Kiezdeutsch as a multiethnolect. In: Kern, Friederike/ Selting, Margret (Hg.): Ethnic styles of speaking in european metropolitan areas. (= Studies in Language Variation 8). Amsterdam/Philadelphia, S. 45-73.

Fürstenau, Susanne/Gogolin, Ingrid/Yağmur, Kutlay (Hg.) (2003): Mehrsprachigkeit in Hamburg. Münster.

Goffman, Erving (1981): Forms of talk. Phildaelphia.

Gogolin, Ingrid (2010): Stichwort: Mehrsprachigkeit. In: Zeitschrift für Erziehungswissenschaft 13, S. 529-547.

Gogolin, Ingrid/Meyer, Meinert A. (2010): Editorial. In: Zeitschrift für Erziehungswissenschaft 13 , S. 525-528.

Haug, Sonja (2008): Sprachliche Integration von Migranten in Deutschland. Working Paper 14 der Forschungsgruppe des Bundesamtes. Nürnberg. Internet: www.bamf. de/SharedDocs/Anlagen/DE/Publikationen/WorkingPapers/wp14-sprachliche-integration. pdf?_blob=publicationFile (Stand: 14.05.2012).

Keim, Inken (2007): Socio-cultural identity, communicative style, and their change over time: a case study of an immigrant youth group in Mannheim, Germany. In: Auer, Peter (Hg.): Style and social identities: alternative approaches to linguistic heterogeneity. (= Language, Power and Social Process 18). Berlin, S. 155-186.

Klieme, Eckhard et al. (Hg.) (2010): PISA 2009. Bilanz nach einem Jahrzehnt. Münster.

Kramsch, Claire (2009): The multilingual subject. What language learners say about their experience and why it matters. Oxford. 
Krüger-Potratz, Marianne (1991): Anderssein gab es nicht. Ausländer und Minderheiten in der DDR. Münster.

Lamarre, Patricia/Lamarre, Stéphanie (2009): Montréal „on the move“: Pour une approche ethnographique non-statique des pratiques langagières des jeunes multilingues. In: Bulot, Thierry (Hg.): Formes \& normes sociolinguistiques. Ségrégations et discriminations urbaines. Paris, S. 105-134.

Liebmann, Irina (2002): Berliner Mietshaus. Berlin.

Mac Giolla Chríost, Diarmait (2007): Language and the city. Basingstoke.

Makoni, Sinfree/Pennycook, Alastair (Hg.) (2007): Disinventing and reconstituting languages. (= Bilingual Education and Bilingualism 62). Cleveland.

Meng, Katharina (2001): Russlanddeutsche Sprachbiografien: Untersuchungen zur sprachlichen Integration von Aussiedlerfamilien. (= Studien zur Deutschen Sprache 21). Tübingen.

Meyer, Bernd (2011): Herkunftssprachen als kommunikative Ressource? In: Eichinger, Ludwig M./Plewnia, Albrecht/Steinle, Melanie (Hg.): Sprache und Integration. Über Mehrsprachigkeit und Migration. (= Studien zur Deutschen Sprache 57). Tübingen, S. 189-213.

Ministerium für Schule und Weiterbildung des Landes Nordrhein-Westfalen (2011): Das Schulwesen in Nordrhein-Westfalen aus quantitativer Sicht 2010/11. Statistische Übersicht 373, 2. Auflage. Düsseldorf. Internet: www.schulministerium.nrw.de/ BP/Schulsystem/Statistik/2010_11/StatUebers373.pdf (Stand: 14.05.2012).

Nekvapil, Jiři (2000): On non-self-evident relationships between language and ethnicity: how Germans do not speak German, and Czechs do not speak Czech. In: Multilingua 19, S. 37-53.

Nekvapil, Jiři (2003): Language biographies and the analysis of language situations: on the life of the German community in the Czech Republic. In: International Journal of the Sociology of Language 162, S. 63-83.

Norton, Bonny (2000): Identity and language learning: gender, ethnicity and educational change. Harlow.

Ohliger, Rainer/Raiser, Ulrich (2005): Integration und Migration in Berlin. Zahlen Daten - Fakten. Berlin. Internet: www.berlin.de/imperia/md/content/lb-integration -migration/publikationen/beitraege/zahlen_daten_fakten_bf.pdf?start\&ts=1277466942\& file=zahlen_daten_fakten_bf.pdf (Stand: 14.05.2012).

Pavlenko, Aneta (2007): Autobiographic narratives as data in applied linguistics. In: Applied Linguistics 28, S. 163-188.

Pennycook, Alastair (2010): Language as a local practice. London.

Pfaff, Carol (2010): Multilingual development in Germany in the crossfire of ideology and politics. In: Okulska, Urszula/Cap, Piotr (Hg.): Perspectives in politics and discourse. (= Discourse Approaches to Politics, Society and Culture 36). Amsterdam, S. 328-357. 
Projektgruppe SPREEG (2001): Was Kinder sprechen! Überlegungen zu einer Sprachenerhebung an Essener Grundschulen. In: ELiSe (Essener Linguistische Skripte elektronisch) 1/2, S. 75-89.

Rampton, Ben (in diesem Band): From ,Youth Language' to contemporary urban vernaculars.

Rockmann, Ulrike (2010): Wer sind und wo leben die Zuwanderer in Berlin? Präsentation für Amt für Statistik Berlin-Brandenburg. Internet: www.berlin.de/imperia/md/ content/lb-integration-migration/statistik/pk_praesentation_afs_bed_bf.pdf?start\&ts=130 7657666\&file=pk_praesentation_afs_bed_bf.pdf (Stand: 14.05.2012).

Schroeder, Christoph (2007): Integration und Sprache. In: Aus Politik und Zeitgeschichte 22-23, S. 6-12.

Statistik Austria (Hg.) (2002): Volkszählung 2001 Hauptergebnisse I - Österreich. Wien.

Statistisches Bundesamt (Hg.) (2006): Datenreport 2006: Zahlen und Fakten über die Bundesrepublik Deutschland. In Zusammenarbeit mit dem Wissenschaftszentrum Berlin für Sozialforschung (WZB) und dem Zentrum für Umfragen, Methoden und Analysen, Mannheim (ZUMA). Bonn. Internet: www.gesis.org/fileadmin/upload/ forschung/publikationen/datenreport/2006/teil2.pdf.

Statistisches Bundesamt (Hg.) (2011): Bevölkerung und Erwerbstätigkeit. Bevölkerung mit Migrationshintergrund - Ergebnisse des Mikrozensus 2010 - Fachserie 1 Reihe 2.2. Wiesbaden. Internet: www.destatis.de/DE/Publikationen/Thematisch/Bevoelkerung /MigrationIntegration/Migrationshintergrund2010220107004.pdf?_blob=publicationFile.

Tophinke, Doris (2002): Lebensgeschichte und Sprache. Zum Konzept der Sprachbiografie aus linguistischer Sicht. In: Bulletin Suisse de Linguistique Appliquée 76, S. $1-14$.

Treichel, Bärbel/Bethge, Katrin (2010): Neue europäische Mehrsprachigkeit. Zum Zusammenhang von Sprache und Biographie in europäischen Lebensgeschichten. In: Zeitschrift für Literaturwissenschaft und Linguistik 160, S. 107-128.

Valentine, Gill/Sporton, Deborah/Bang Nielsen, Katrine (2009): The spaces of language: the everyday practices of young Somali refugees and asylum seekers. In: Collins, James/Slembrouck, Stef/Baynham, Mike (Hg.): Globalization and language in contact: Scale, migration and communicative practices. London, S. 189-206.

Vertovec, Steven (2007): Super-diversity and its implications. In: Ethnic and Racial Studies 30, S. 1024-1054.

Vertovec, Steven (2010): Towards post-multiculturalism? Changing communities, conditions and contexts of diversity. In: International Journal of Social Science 6/199, S. 83-95.

Wiese, Heike (in diesem Band): Das Potenzial multiethnischer Sprechergemeinschaften.

Weiss, Karin/Dennis, Mike (Hg.) (2005): Erfolg in der Nische? Die Vietnamesen in der DDR und Ostdeutschland. (= Studien zu Migration und Minderheiten 13). Berlin. 
